\title{
ALDH1 is an independent prognostic factor for patients with stages II-III rectal cancer after receiving radiochemotherapy
}

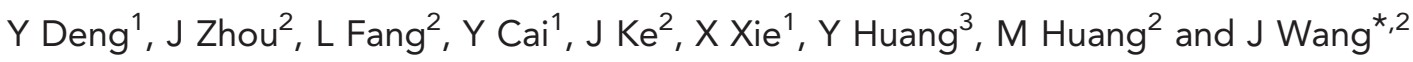 \\ ${ }^{1}$ Department of Medical Oncology, The Sixth Affiliated Hospital, Sun Yat-sen University, Guangzhou, China; ${ }^{2}$ Department \\ of Colorectal Surgery, The Sixth Affiliated Hospital, Sun Yat-sen University, Guangzhou, China and ${ }^{3}$ Department of Pathology, \\ The Sixth Affiliated Hospital, Sun Yat-sen University, Guangzhou, China
}

Background: About one in five patients with locally advanced rectal cancer (RC) suffers recurrence or distant metastasis after neoadjuvant therapy. We investigated how cancer stem cell markers change after neoadjuvant therapy and how these markers relate to recurrence.

Methods: Pretreatment biopsies and postoperative specimens were taken from 64 patients with locally advanced rectal adenocarcinoma who received preoperative radiochemotherapy (RCT) between sampling. Samples were tested immunohistochemically for CD44, LGR5, ALDH1 and CD166; scores were dichotomised as high or low. The median follow-up period was 36 months.

Results: High expression of CD44, LGR5, ALDH and CD166 was found in 38\%,5\%,48\% and 10\%, respectively, before RCT and $86 \%, 33 \%, 71 \%$ and $52 \%$, respectively, after RCT. CD44 $(P=0.001)$, LGR5 $(P=0.049)$ and CD166 $(P=0.003)$ were significantly upregulated after RCT. Whereas no recurrence was seen during the follow-up in the low ALDH group, 40\% of the high ALDH group suffered recurrence. In multivariate COX analysis, postoperative ALDH1 independently predicted poor prognosis in patients with RC who received RCT $(P=0.0095)$.

Conclusion: Preoperative RCT upregulates expression of stem cell markers in patients with RC. High post-treatment ALDH1 expression predicts poor prognosis for these patients after neoadjuvant therapy.

Mounting evidence indicates that human cancers derive from cancer-initiating cells or 'cancer stem cells' (CSCs) (O'Brien et al, 2007; Ricci-Vitiani et al, 2007). As CSCs can initiate and sustain malignant growth and resist chemo- and radiotherapies (Boman and Wicha, 2008), potential CSC biomarkers have been studied to better understand mechanisms underlying tumour growth, progression and treatment responses (Lugli et al, 2010; Avoranta et al, 2013).

Neoadjuvant radiochemotherapy (RCT) followed by total mesorectal excision (TME) has become the standard treatment for locally advanced stages II-III rectal cancer (RC) (using the American Joint Committee on Cancer (AJCC) guidelines) within the last decade (Sauer et al, 2004). Individual responses, however, vary considerably (Rodel et al, 2005). Cytotoxic agents are thought to reduce tumour volume by killing actively proliferating cells but sparing some CSCs, which acquire resistance from these therapies through natural selection and genetic mutation. Reportedly, enrichment of the stem cell marker CD133 in RC treated with preoperative RCT independently predicts metastasis and poor survival (Sprenger et al, 2013). CD133 was initially identified as a reliable CSC marker in primary human colorectal cancer (CRC; O’Brien et al, 2007; Ricci-Vitiani et al, 2007); however, this use is controversial (Shmelkov et al, 2008). Alternatively, CD44, LGR5 (Kemper et al, 2012), CD166 and ALDH molecules 
(among others) have also been reported as CSC markers in CRC (Lugli et al, 2010).

In this study, expression of several CRC stem cell markers CD44, LGR5, ALDH and CD166 - were evaluated, for the first time, synchronously in both pretreatment biopsies and corresponding surgical specimens from patients with locally advanced RC who were treated with standardised preoperative RCT. Expression levels of markers were correlated with clinicopathologic characteristics and clinical follow-up.

\section{PATIENTS AND METHODS}

Patient cohort. Sixty-four patients with locally advanced AJCC stages II-III rectal adenocarcinoma treated between August 2009 and January 2011 were included in the study - 21 patients in the discovery set and another 43 in the validation set. All patients underwent standardised preoperative RCT and quality-assessed curative TME surgery in The Sixth Affiliated Hospital of Sun Yatsen University. cTNM classifications were determined using the AJCC guidelines. This study was approved by the Medical Ethics Committee of The Sixth Affiliated Hospital of Sun Yat-sen University. All patients gave informed consent for use of biopsies and tumour samples. Staging procedures, including colonoscopy, contrast-enhanced CT scans of the thorax, abdomen and pelvis and pelvic MRIs, were performed in all cases to confirm locally advanced tumour stage and to exclude patients with evidence of distant metastatic disease at the initial RC diagnosis.

Treatment procedures, pathological-staging procedures and tumour regression grading. Treatment consisted of neoadjuvant RCT. Each patient received a total irradiation dose of $46 \mathrm{~Gy}$ in 23 fractions of $2 \mathrm{~Gy}$ with concomitant application of 5-fluorouracil (5-FU). Surgical procedures comprised anterior resections, abdominoperineal resections and discontinuous resections (Hartmann procedure). Perioperative assessment of TME quality was performed in all surgical specimens. Pretreatment biopsy material was taken endoscopically. Pathological staging of post-treatment tumour resections was performed using AJCC 7th TNM classifications. Histopathological tumour regression grade (TRG) was based on a semiquantitative 4-point grading system described by the NCCN guidelines: Grade 0 (complete response): no viable cancer cells; Grade 1 (moderate response): single cells or small groups of cancer cells; Grade 2 (minimal response): residual cancer outgrown by fibrosis; Grade 3 (poor response): minimal or no tumour kill; extensive residual cancer (Ryan et al, 2005). Patients whose preoperative biopsies were too small or who had too few postoperative cancer cells (TRG 0/1) were excluded from the study.

Immunohistochemical staining. All matched pre- and postsurgical samples were fixed in $10 \%$ buffered formalin and embedded in paraffin. Sections were cut at $3-\mu \mathrm{m}$ thickness and mounted on APES-coated glass slides. Slides were deparaffinised in xylene three times for $15 \mathrm{~min}$, rehydrated through graded ethanol for a total of $25 \mathrm{~min}$ and immersed in distilled water for $1 \mathrm{~min}$. To retrieve antigens, slides were heated in $0.01 \mathrm{M}$ citrate buffer (pH 6.0) in a microwave oven at $100^{\circ} \mathrm{C}$ for $5 \mathrm{~min}$ and at $40^{\circ} \mathrm{C}$ for $10 \mathrm{~min}$. Slides were then immersed in phosphate-buffered solution (PBS) for $5 \mathrm{~min}$, twice. Sections were incubated with the following primary antibodies: anti-human CD166 (clone M0G/07; 1:200; Novocastra, Newcastle, UK), anti-human CD44s (clone DF1485; 1:50; Dako, Glostrup, Denmark), anti-human lgr5 (polyclonal; 1:1000; Abcam, Cambridge, UK) and anti-human ALDH1 isoforma1 (polyclonal; 1:500; Abcam). Negative controls underwent the same protocol without primary antibodies in a humidified chamber and were refrigerated at $4{ }^{\circ} \mathrm{C}$ overnight. Slides were then rinsed with $\mathrm{PBS}$ three times and incubated with polyperoxidaseanti-mouse/rabbit IgG (Zymed, San Diego, CA, USA) for $15 \mathrm{~min}$.
After washing with PBS, 3,3'-diaminobenzidine was used as the chromogen. Sections were counterstained for $2 \mathrm{~min}$ with hematoxylin solution.

Preparation of the tissue microarray (TMA). Postoperative tumour tissue and adjacent mucosa were studied. Paraffin blocks containing areas of invasive carcinoma were identified on corresponding $\mathrm{H}$ - and E-stained sections. Areas of interest that represented the invasive front of the tumour, rich in non-necrotic tumoral glands, were identified and marked on the source block. The source block was then cored and a 1-mm core transferred to the recipient block using the Beecher Tissue Microarray (Beecher Instruments, Silver Spring, MD, USA). Tow cores were arrayed per specimen. Adjacent mucosa was taken from the proximal or distal stump.

Immunohistochemical (IHC) scoring. Immunostaining was evaluated by two independent pathologists blinded to the study. Expression was analysed by an individual labeling score considering percent of positive cells and staining intensity. Intensity was scored as 0 , none; 1 , weak; 2 , moderate; 3 , strong; and 4 , extremely strong. To dichotomise the markers, 0 and 1 were defined as low expression, and 3 and 4 as high expression. The fraction of positive tumour cells was assigned to 0 (no staining), 1 ( $<25 \%$ positive), 2 ( $25-50 \%$ positive), 3 (50-75\% positive) and 4 ( $>75 \%$ positive). Intensity and fraction of positive cell scores were multiplied for each marker and thus ranged from 0 (no positive cells) to 16 ( $>75 \%$ of tumour cells with intense staining). To dichotomise the markers, optimal cutoff points were calculated using the SPSS 17.0 ROC curve. TMA scores were obtained by the TMAJ software (JHU Tissue MicroArray Core Facility, Baltimore, MA, USA).

Clinical evaluation and follow-up. All patients were re-evaluated at 3-month intervals for 2 years and every 6 months thereafter. Evaluations consisted of pertinent medical history, physical examination, blood cell counts and blood chemistry including carcinoembryonic antigen (CEA) levels at every follow-up visit. Colonoscopies (in patients treated with anterior resections) were performed at 6-month intervals in the first 2 years, and annually thereafter. Follow-up chest, abdomen and pelvis CT studies were scheduled every 6 months for the first 3 years, and annually thereafter. Histological confirmation of local recurrence and distant relapse (defined as tumour manifestation outside the pelvis) was encouraged. Alternate acceptable criteria included sequential enlargement of a mass in radiologic studies with simultaneous increase in serum CEA levels. Disease-free survival (DFS) was defined as the time from surgery to detection of any tumour relapse. The median follow-up time was 36 months (range: 24-40 months).

Statistical analysis. Statistical analysis used SPSS 17.0. Frequency tables were analysed using the $\chi^{2}$ test, with the likelihood ratio (LR) or Fisher's exact test for categorical variables. Pairwise comparison of expression in biopsies and operative samples was analysed using a nonparametric paired-samples test (Wilcoxon signed-ranks test). Correlation of markers' labeling scores with clinical outcome variables was computed by Spearman rank correlation. The ROC curve was used to set the cutoff point. Time to event data were visualised using Kaplan-Meier analysis with log-rank test. $P \leqslant 0.05$ was considered significant for all tests.

\section{RESULTS}

Clinical results and cancer relapse. Table 1 shows patients' clinicopathological characteristics. In initial pretreatment staging, 51 of the 64 patients had mesorectal lymph node involvement. After preoperative RCT, 21 patients had evidence of residual lymph node metastasis. Pathological lymph node staging was based 
on a median lymph node yield of 15 . Positive circumferential margin of resection was not present. Forty-two patients (65.6\%) were downstaged after RCT.

Stem cell marker expression in pre- and post-RCT specimens. CD44, LGR5, ALDH and CD166 immunoreactions were assessed in all investigated tumour biopsies and corresponding surgical specimens in discovery set patients. Both biopsies and post-treatment

Table 1. Clinicopathological characteristics

\begin{tabular}{|l|c|c|}
\hline Feature & Number & $\%$ \\
\hline Age (years) & \multicolumn{2}{|l|}{} \\
\hline Mean & 58.1 & \\
Median & 57 & \\
Range & $31-82$ & \\
\hline Gender & \multicolumn{2}{|}{} \\
\hline Male & 36 & 56 \\
Female & 28 & 44 \\
\hline Differentiation & \multicolumn{2}{|l|}{} \\
\hline Well & 9 & 14 \\
Moderate & 41 & 60 \\
Poor & 4 & 6 \\
\hline
\end{tabular}

Tumour anal verge distance $(\mathrm{cm})$

\begin{tabular}{|l|l|l|}
\hline $0-6$ & 45 & 70 \\
$>6-12$ & 19 & 30 \\
\hline
\end{tabular}

cT stage

\begin{tabular}{|l|c|c|}
\hline 2 & 9 & 14 \\
3 & 45 & 70 \\
4 & 10 & 16 \\
\hline
\end{tabular}

\section{$\mathrm{cN}$ stage}

Positive

Negative

\begin{tabular}{l|l}
51 & 80
\end{tabular}

\section{Resection status}

\begin{tabular}{|c|c|c|}
\hline R0 & 64 & 100 \\
R1 & 0 & 0 \\
R2 & 0 & 0 \\
\hline
\end{tabular}

\section{CRM}

Positive

Negative

Tumour regression grading

\begin{tabular}{|c|c|c|}
\hline $\begin{array}{l}1 \\
2 \\
3\end{array}$ & $\begin{array}{l}16 \\
21 \\
27\end{array}$ & $\begin{array}{l}25 \\
33 \\
42\end{array}$ \\
\hline \multicolumn{3}{|c|}{ ypT stage } \\
\hline $\begin{array}{l}1 \\
2 \\
3 \\
4\end{array}$ & $\begin{array}{c}7 \\
9 \\
45 \\
3\end{array}$ & $\begin{array}{c}11 \\
14 \\
70 \\
5\end{array}$ \\
\hline \multicolumn{3}{|c|}{ ypN stage } \\
\hline $\begin{array}{l}0 \\
1 \\
2\end{array}$ & $\begin{array}{c}42 \\
18 \\
4\end{array}$ & $\begin{array}{c}66 \\
28 \\
6\end{array}$ \\
\hline
\end{tabular}

tumour samples showed predominately homogeneous positivity. Table 2 shows immunopositive percentages for the four markers by the dichotomised scores. High expression rates for CD44, LGR5, ALDH and CD166 were 38\%,5\%,48\% and $10 \%$, respectively, before neoadjuvant therapy, and $86 \%(P=0.001), 33 \%(P=0.049)$, $71 \%(P=0.116)$ and $52 \%(P=0.003)$, respectively, afterwards (Table 2). Expressions of CD44, LGR5 and CD166 were significantly upregulated after neoadjuvant RCT (Figure 1). CD44 was upregulated in eight patients, and downregulated in none; six and nine patients had upregulated LGR5 and CD166 expression, respectively. Immunohistochemical pictures are shown in Supplementary Figure 1S.

Initial stem cell marker expression, clinicopathological parameters and survival. In pre-RCT specimens, CD44, LGR5, ALDH1 and CD166 expressions did not correlate to each other. No clinicopathologic parameters correlated with CD44, LGR5, ALDH1 or CD166 expression. Preoperative high or low CD44, ALDH1 and CD166 expression did not predict DFS (Supplementary Figure 2S). No analysis was performed for LGR5 as only one patient showed as positive.

Post-therapeutic stem cell marker expression, clinicopathological parameters and survival. No clinicopathological parameters correlated to CD44, LGR5, ALDH1 or CD166 expression levels postoperatively. No markers except ALDH1 were related to survival. Among 15 patients with high ALDH1 expression, six showed distant metastases or local recurrence (40\%) within the follow-up period, whereas no patient with low ALDH1 expression developed metastasis or local recurrence; this implies that ALDH1 expression distinguishes patients at high risk for relapse. As ALDH1 expression after RCT predicted relapse in Kaplan-Meier analysis $(P=0.041$, Supplementary Figure $3 S)$, we analysed ALDH1 in a validation data set. High ALDH1 expression was inversely associated with advanced downstaging $(P=0.010)$. In downstaged patients (sensitive group), ALDH1 expression rate was $50 \%$ but was $100 \%$ in non-downstaged patients (resistant group) $(P=0.019)$.

Postoperative ALDH1 expression independently predicted tumour recurrence. ALDH1 expression in 43 patients' tissue was postoperatively studied using TMA. The IHC scores

Table 2. Expression of CD44, Lgr5, ALDH and CD166 before and after radiochemotherapy

\begin{tabular}{|c|c|c|c|}
\hline & \multicolumn{2}{|c|}{ No. of patients (\%) } & \multirow[b]{2}{*}{$\boldsymbol{P}$-value } \\
\hline & Biopsy & Tumour & \\
\hline \multicolumn{4}{|c|}{ CD44 } \\
\hline $\begin{array}{l}\text { High } \\
\text { Low }\end{array}$ & $\begin{array}{r}8(38) \\
13(62)\end{array}$ & $\begin{array}{r}18(86) \\
3(14)\end{array}$ & 0.001 \\
\hline \multicolumn{4}{|l|}{ Lgr5 } \\
\hline $\begin{array}{l}\text { High } \\
\text { Low }\end{array}$ & $\begin{array}{c}1(5) \\
20(95)\end{array}$ & $\begin{array}{r}7(33) \\
14(67)\end{array}$ & 0.049 \\
\hline \multicolumn{4}{|c|}{ ALDH } \\
\hline $\begin{array}{l}\text { High } \\
\text { Low }\end{array}$ & $\begin{array}{l}10(48) \\
11(52)\end{array}$ & $\begin{array}{r}15(71) \\
6(29)\end{array}$ & 0.116 \\
\hline \multicolumn{4}{|c|}{ CD166 } \\
\hline $\begin{array}{l}\text { High } \\
\text { Low }\end{array}$ & $\begin{array}{r}2(10) \\
19(90)\end{array}$ & $\begin{array}{l}11(52) \\
10(48)\end{array}$ & 0.003 \\
\hline
\end{tabular}



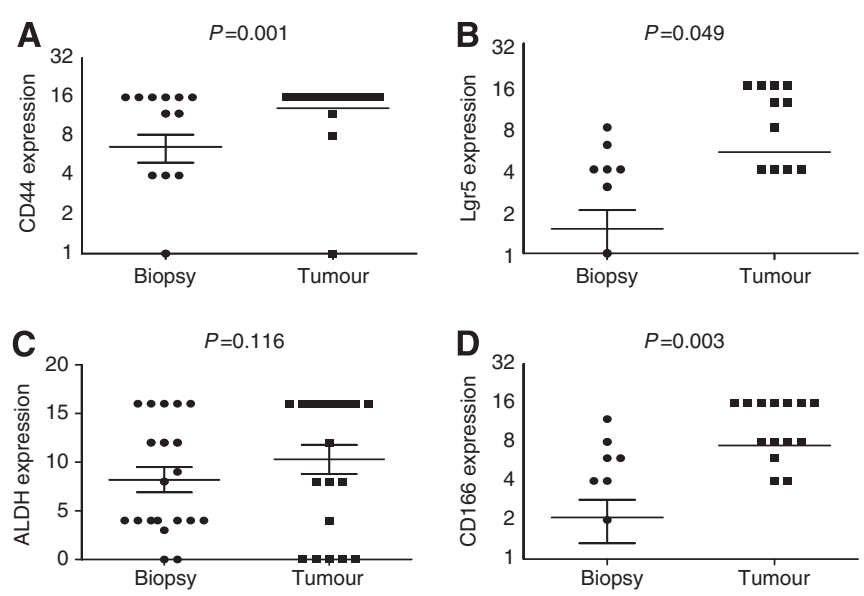

Figure 1. Increased expression of CD44 (A), Lgr5 (B), and CD166 (D) after preoperative radiochemotherapy. Expression scores were calculated by multiplying positive intensity and percentage in immunostained samples. No change in ALDH (C).

(consecutive variable) were obtained through the TMAJ software, with a median score of 14.83 (range: 0.49-96.67). When postoperative ALDH1, age, sex, cTNM, preoperative CEA, ypTNM and TRG were tested as prognostic factors with multivariate COX regression, only ALDH1 $(P=0.0095)$ and ypTNM $(P=0.0015)$ were found to significantly affect DFS in patients who received RCT. We used Student's $t$-test to exclude the interaction between ALDH1 and ypTNM; the results showed that there was no correlation between the two factors $(t=0.48, P=0.636)$. After adjusting for other factors, recurrence risk for stage III patients was 9.99 times higher than that for stage I/II patients. Recurrence risk increased $0.03 \%$ for each point increase in ALDH1 score. In a ROC curve for the prognostic sensitivity and specificity of ALDH1, the area under curve was 0.796 Supplementary Figure 4S) and the optimal cutoff was 21.4. By using this cutoff in a Kaplan-Meier analysis, we found that the survival curve between the two groups was separated significantly $(P=0.009$; Figure 2$)$. Postoperative high ALDH1 expression was an independent predictor of poor prognosis for RC patients after RCT.

\section{DISCUSSION}

Recent evidence suggests that CRC CSCs can renew themselves and are relatively insensitive to chemotherapy, which is targeted at homogeneous populations of rapidly growing and underdifferentiated tumour cells (Boman and Wicha, 2008). Our previous in vitro study showed that CD133 expression was upregulated and cells became more 'stemlike' after 5-FU exposure (Deng et al, 2010). Dylla et al (2008) found that CRC CSCs were enriched in colon tumours after chemotherapy and could rapidly regenerate their tumours of origin in a xenograft model. However, few such studies investigated CSCs in living human patients.

Here, we compared expression of several CSC markers in matched pretreatment biopsies and corresponding surgical specimens of 21 patients with locally advanced RC who were treated with standardised 5-FU-based neoadjuvant RCT. In comparing pretreatment biopsies and surgical resection specimens, we saw significant upregulation of CD44, CD166 and LGR5 in cancer cells following RCT. This concords with findings of significant upregulation of CSC expression after treatment with neoadjuvant chemotherapy or RCT (Iinuma et al, 2011). Expression of another stem cell marker, ALDH1, increased in four patients whose biopsies were initially ALDH1. ALDH1 expression trended

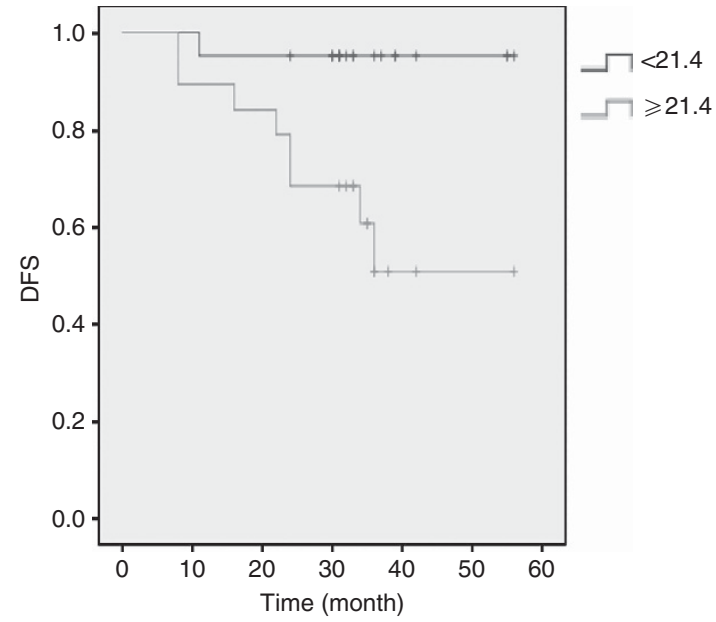

Figure 2. Patients with high post-therapy ALDH1 expression ( $\geqslant 21.4$ in TMAJ score) had shorter disease-free survival in Kaplan-Meier analysis $(P=0.009)$.

upwards after RCT, although not significantly, and was significantly higher in resistant patients (100\%) compared with chemosensitive patients $(50 \%)$. We found that tumour cells had upregulated expression of stem cell markers during RCT in RC, indicating that RCT spares CSCs or enriched cells with stem cell phenotypes in RC patients who received neoadjuvant therapy. Initial stem cell markers did not correlate to the regression of tumour grade and prognosis, possibly because CSC counterparts in stage II-III patients are a small population. However, as residual stem cells escape therapy, they have more chances to gain epithelial-mesenchymal transformation and cause metastases.

Our present investigation found that high ALDH1 expression in post-treatment specimens was an independent predictor of recurrence in RC patients who receive neoadjuvant RCT. ALDH1 was strongly predictive in a recent investigation (Rodel et al, 2005). Accordingly, the current study demonstrates that high posttreatment ALDH expression significantly predicts cancer relapse and distant metastases, with recurrence risk increasing by $0.03 \%$ for each point increase in ALDH1 expression. This also underscores the potential role of ALDH1 in metastatic pathways. ALDH1 is a detoxifying enzyme that oxidises intracellular aldehydes and thereby confers resistance to alkylating agents (Dylla et al, 2008). Possibly, the detoxification capacity of ALDH1 protects CSCs against oxidative insult, thus supporting their wellrecognised longevity. ALDH-based markers can be used to track colonic stem cells during tumorigenesis. Reportedly, ALDH1 + cells indicate increasing colonic CSC numbers as CRC progresses (Huang et al, 2009). Huang et al (2009) recently showed that $\mathrm{CD} 133+$ and CD44 + cells identified subpopulations that were seven-fold larger than the ALDH + population, within normal colonic tissues, and concluded that the ALDH1 + CSCs are a subset of CD44 + CSCs. This subset of stem cells could be insensitive to chemical or radiation therapy. ALDH1 enzymatic activity is a major mediator of resistance to cyclophosphamide - a classical chemotherapeutic agent (Dylla et al, 2008). ALDH1 is now widely accepted as a CRC-CSC marker (Arcaroli et al, 2012; Shenoy et al, 2012). However, only high residual ALDH1 expression after RCT significantly predicted distant metastasis or local recurrence. Among patients with high ALDH1 expression, $40 \%$ had recurrent cancer, whereas no patients with low ALDH1 expression did. Residual high expression, rather than initial or increased ALDH1 expression, was highly related to poor prognosis. We recommend conducting ALDH1 staining routinely for patients who receive neoadjuvant RCT to better predict the recurrence risk. 
More effective adjuvant treatment should also be investigated for those patients with high ALDH1 expression.

In summary, our results show that therapeutic strategies upregulate expression of stem cell markers CD44, LGR5 and CD166 during the preoperative therapy in RC patients. High postoperative ALDH1 expression predicts poor prognosis for CRC patients who received neoadjuvant therapy. However, the implications of ALDH1 expression in RC should be validated in a clinical trial.

\section{ACKNOWLEDGEMENTS}

The study was supported by the National Natural Science Foundation (No. 81101669).

\section{CONFLICT OF INTEREST}

The authors declare no conflicts of interest.

\section{REFERENCES}

Arcaroli JJ, Powell RW, Varella-Garcia M, McManus M, Tan AC, Quackenbush KS, Pitts TM, Gao D, Spreafico A, Dasari A, Touban BM, Messersmith WA (2012) ALDH + tumor-initiating cells exhibiting gain in NOTCH1 gene copy number have enhanced regrowth sensitivity to a gamma-secretase inhibitor and irinotecan in colorectal cancer. Mol Oncol 6: $370-381$.

Avoranta ST, Korkeila EA, Ristamaki RH, Syrjanen KJ, Carpen OM, Pyrhonen SO, Sundstrom JT (2013) ALDH1 expression indicates chemotherapy resistance and poor outcome in node-negative rectal cancer. Hum Pathol 44: 966-974.

Boman BM, Wicha MS (2008) Cancer stem cells: a step toward the cure. J Clin Oncol 26: 2795-2799.

Deng YH, Pu XX, Huang MJ, Xiao J, Zhou JM, Lin TY, Lin EH (2010) 5 -Fluorouracil upregulates the activity of Wnt signaling pathway in CD133-positive colon cancer stem-like cells. Chin J Cancer 29: 810-815.

Dylla SJ, Beviglia L, Park IK, Chartier C, Raval J, Ngan L, Pickell K, Aguilar J, Lazetic S, Smith-Berdan S, Clarke MF, Hoey T, Lewicki J, Gurney AL (2008) Colorectal cancer stem cells are enriched in xenogeneic tumors following chemotherapy. PLoS One 3: e2428.

Huang EH, Hynes MJ, Zhang T, Ginestier C, Dontu G, Appelman H, Fields JZ, Wicha MS, Boman BM (2009) Aldehyde dehydrogenase 1 is a marker for normal and malignant human colonic stem cells (SC) and tracks SC overpopulation during colon tumorigenesis. Cancer Res 69: 3382-3389.
Iinuma H, Watanabe T, Mimori K, Adachi M, Hayashi N, Tamura J, Matsuda K, Fukushima R, Okinaga K, Sasako M, Mori M (2011) Clinical significance of circulating tumor cells, including cancer stem-like cells, in peripheral blood for recurrence and prognosis in patients with Dukes' stage B and C colorectal cancer. J Clin Oncol 29: 1547-1555.

Kemper K, Prasetyanti PR, De Lau W, Rodermond H, Clevers H, Medema JP (2012) Monoclonal antibodies against Lgr5 identify human colorectal cancer stem cells. Stem Cells 30: 2378-2386.

Lugli A, Iezzi G, Hostettler I, Muraro MG, Mele V, Tornillo L, Carafa V, Spagnoli G, Terracciano L, Zlobec I (2010) Prognostic impact of the expression of putative cancer stem cell markers CD133, CD166, CD44s, EpCAM, and ALDH1 in colorectal cancer. Br J Cancer 103: 382-390.

O’Brien CA, Pollett A, Gallinger S, Dick JE (2007) A human colon cancer cell capable of initiating tumour growth in immunodeficient mice. Nature $\mathbf{4 4 5}$ : 106-110.

Ricci-Vitiani L, Lombardi DG, Pilozzi E, Biffoni M, Todaro M, Peschle C, De Maria R (2007) Identification and expansion of human colon-cancerinitiating cells. Nature 445: 111-115.

Rodel C, Martus P, Papadoupolos T, Fuzesi L, Klimpfinger M, Fietkau R, Liersch T, Hohenberger W, Raab R, Sauer R, Wittekind C (2005) Prognostic significance of tumor regression after preoperative chemoradiotherapy for rectal cancer. J Clin Oncol 23: 8688-8696.

Ryan R, Gibbons D, Hyland JM, Treanor D, White A, Mulcahy HE, O’Donoghue DP, Moriarty M, Fennelly D, Sheahan K (2005) Pathological response following long-course neoadjuvant chemoradiotherapy for locally advanced rectal cancer. Histopathology 47: 141-146.

Sauer R, Becker H, Hohenberger W, Rodel C, Wittekind C, Fietkau R, Martus P, Tschmelitsch J, Hager E, Hess CF, Karstens JH, Liersch T, Schmidberger H, Raab R (2004) Preoperative versus postoperative chemoradiotherapy for rectal cancer. $N$ Engl J Med 351: 1731-1740.

Shenoy AK, Fisher RC, Butterworth EA, Pi L, Chang LJ, Appelman HD, Chang M, Scott EW, Huang EH (2012) Transition from colitis to cancer: high Wnt activity sustains the tumor-initiating potential of colon cancer stem cell precursors. Cancer Res 72: 5091-5100.

Shmelkov SV, Butler JM, Hooper AT, Hormigo A, Kushner J, Milde T, St Clair R, Baljevic M, White I, Jin DK, Chadburn A, Murphy AJ, Valenzuela DM, Gale NW, Thurston G, Yancopoulos GD, D’Angelica M, Kemeny N, Lyden D, Rafii S (2008) CD133 expression is not restricted to stem cells, and both CD133 + and CD133- metastatic colon cancer cells initiate tumors. J Clin Invest 118: 2111-2120.

Sprenger T, Conradi LC, Beissbarth T, Ermert H, Homayounfar K, Middel P, Ruschoff J, Wolff HA, Schuler P, Ghadimi BM, Rodel C, Becker H, Rodel F, Liersch T (2013) Enrichment of CD133-expressing cells in rectal cancers treated with preoperative radiochemotherapy is an independent marker for metastasis and survival. Cancer 119: 26-35.

This work is published under the standard license to publish agreement. After 12 months the work will become freely available and the license terms will switch to a Creative Commons AttributionNonCommercial-Share Alike 3.0 Unported License.

Supplementary Information accompanies this paper on British Journal of Cancer website (http://www.nature.com/bjc) 